\title{
Newborn with Prenatally Diagnosed Choroidal Fissure Cyst and Panhypopituitarism and Review of the Literature
}

\author{
Ritu Chitkara, M.D., 1,6 Anand Rajani, M.D., 1,6 Jonathan Bernstein, M.D., 2,6 \\ Sejal Shah, M.D., 3,6 Jin S. Hahn, M.D., 4,6 Patrick Barnes, M.D., 5,6 \\ and Susan R. Hintz, M.D., M.S. (Epi.) $)^{1,6}$
}

Little has been reported on fetal diagnosis of choroidal fissure cysts and prediction of the clinical complications that can result. We describe the case of a near-term male infant with prenatally diagnosed choroidal fissure cyst and bilateral clubfeet. His prolonged course in the neonatal intensive care nursery was marked by severe panhypopituitarism, late-onset diabetes insipidus, placement of a cystoperitoneal shunt, and episodes of sepsis. Postnatal genetic evaluation also revealed an interstitial deletion involving most of band 10q26.12 and the proximal half of band 10q26.13. The patient had multiple readmissions for medical and surgical indications and died at 6 months of age. This case represents the severe end of the spectrum of medical complications for children with choroidal fissure cysts. It highlights not only the importance of comprehensive evaluation and multidisciplinary management and counseling in such cases, but also the need for heightened vigilance in these patients.

KEYWORDS: Choroidal fissure cyst, panhypopituitarism, neonate

The choroidal fissure is a space containing cerebrospinal fluid between the fimbria of the hippocampus and the diencephalon. ${ }^{1}$ Choroidal fissure cysts are usually an incidental finding and often considered to be clinically benign, although some have been reported in association with medical and surgical morbidities. ${ }^{1,2}$ However, the range of outcomes is broad, and there is little in the literature to assist in prediction of postnatal course from prenatal findings or to guide postnatal management. We describe a case of prenatal diagnosis of a large choroidal fissure cyst, subsequent difficult neonatal intensive care course including panhypopituitarism and diabetes insipidus, and identification of an interstitial microdeletion of chromosome 10q26. To our knowledge, this constellation of findings has not previously been reported.

\section{CASE REPORT}

The patient was a male infant, born at 37 weeks' estimated gestational age (EGA), with fetal findings of a large choroidal fissure cyst, bilateral clubfeet, and
Divisions of ${ }^{1}$ Neonatology; ${ }^{2}$ Medical Genetics; ${ }^{3}$ Endocrinology; ${ }^{4}$ Neurology and ${ }^{5}$ Radiology; ${ }^{6}$ Department of Pediatrics, Lucile Packard Children's Hospital, Stanford University, Palo Alto, California.

Address for correspondence and reprint requests: Ritu Chitkara, M.D., Fellow in Neonatal-Perinatal Medicine, Division of Neonatology, Department of Pediatrics, Stanford University School of Medicine, 750 Welch Road, Suite 315, Palo Alto, CA 94304 (e-mail: chitkara@stanford.edu).
Am J Perinatol Rep 2011;1:111-114. Copyright (C) 2011 by Thieme Medical Publishers, Inc., 333 Seventh Avenue, New York, NY 10001, USA. Tel: +1 (212) 584-4662.

Received: April 20, 2011. Accepted after revision: July 5, 2011. Published online: November 10, 2011.

DOI: http://dx.doi.org/10.1055/s-0031-1293512.

ISSN 2157-6998. 
A
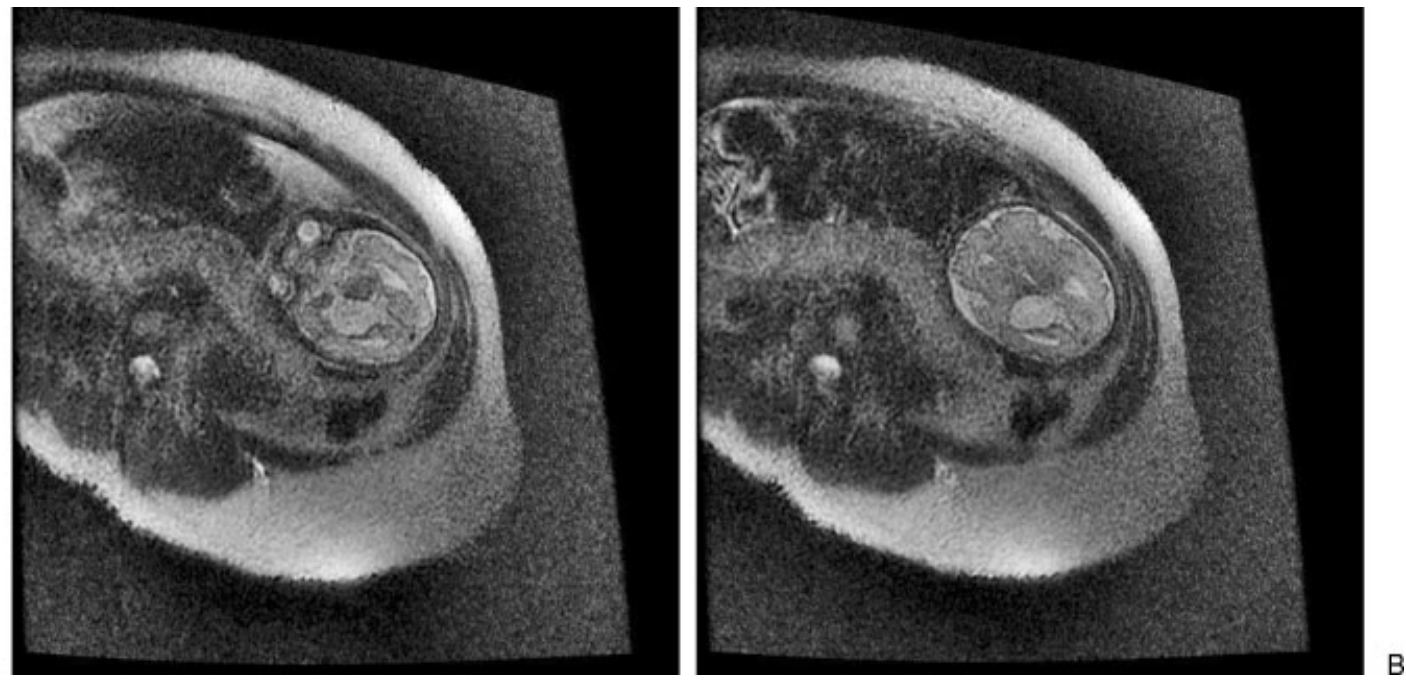

Figure 1 (A, B) Axial T2 single-shot fast spin echo images show fetus at 31 weeks with intracranial cyst in the choroidal fissure.

positive prenatal screen. The expectant mother was referred to our center at 19 weeks' EGA after prenatal screen showed increased risk for trisomy 18 (1/9) and Smith-Lemli-Opitz syndrome (SLOS) (>1/10); level 2 fetal ultrasound revealed an intracranial cyst. Fetal echocardiogram was normal. She received genetic and multidisciplinary counseling and was offered amniocentesis as well as early fetal magnetic resonance imaging (MRI) to further delineate brain anomalies. She declined both, but agreed to a fetal MRI later in pregnancy, which was performed at 31 weeks' EGA. This showed a large rightsided choroidal fissure cyst (28 $\mathrm{mm}$ in diameter) and asymmetric lateral ventricles. The cyst appeared to uplift the undersurface of the left lateral ventricular system. There was evidence of dilation of the frontal horn of the left lateral ventricle. The corpus callosum was noted only anteriorly (Fig. 1A, 1B).
Labor was induced at 37 weeks' EGA due to preeclampsia. The delivery course was unremarkable. The male infant was admitted to the neonatal intensive care unit (NICU). Initial physical exam was notable for a fixed and dilated right pupil, bilateral clubfeet, and micropenis (1.5 cm stretched length). He had a wellshaped cranium, normally set eyes with short palpebral fissures, well-formed and midline nose, and normal palmar as well as phalangeal creases. The left hand was clenched without overlapping fingers. Consultations with genetics, neurology, neurosurgery, ophthalmology, endocrinology and orthopedic surgery departments were obtained during the NICU course. Brain MRI confirmed a large $(3.3 \times 4.8 \mathrm{~cm})$ extraventricular cystic lesion in the right cerebral hemisphere causing mass effect and midline shift with deformity of the thalamomesencephalic region (Figs. 2A, 2B and $3 \mathrm{~A}-\mathrm{C}$ ). $\mathrm{He}$
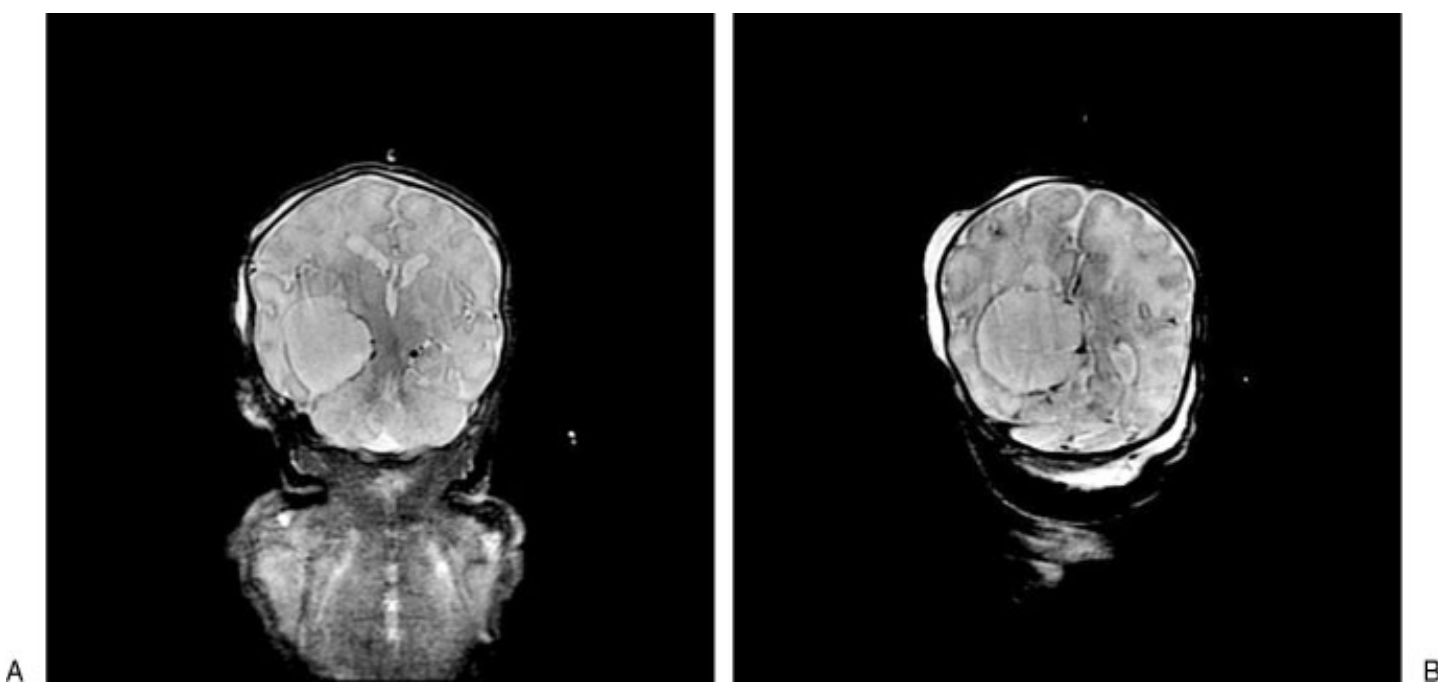

Figure 2 Anterior (A) and posterior (B) coronal T2 (short T1 inversion recovery) images demonstrate cyst extending from midline quadrigeminal plate cistern to choroidal fissure, medial to right ventricular trigone, to temporal horn. 
A
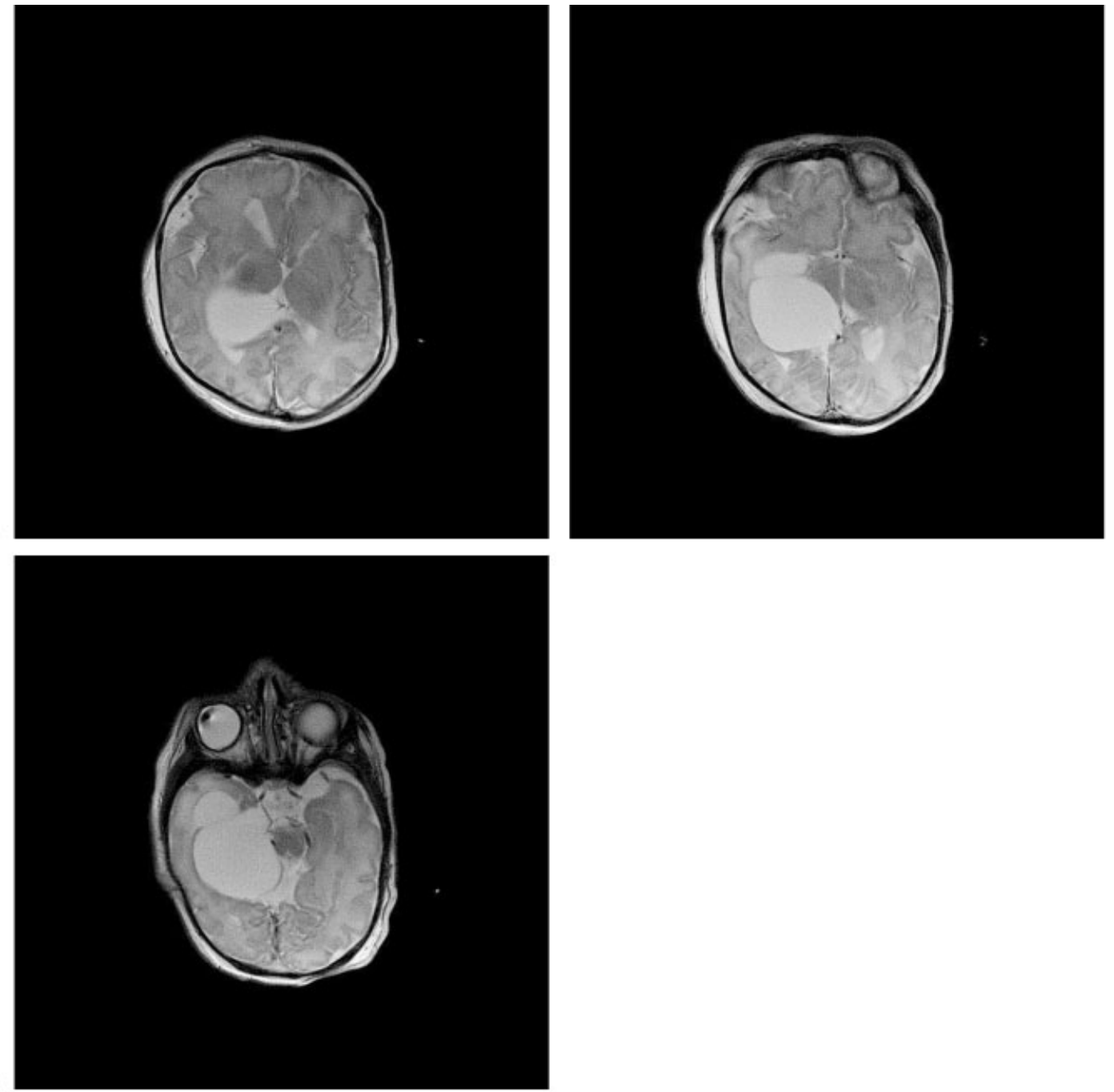

Figure 3 (A-C) Axial neonatal magnetic resonance images demonstrating choroidal fissure cyst.

underwent cyst fenestration and cystoperitoneal shunt placement on day of life 7 without significant initial cyst decompression. Chromosomal analysis showed 46,XY and normal 7-deoxycholesterol levels, excluding SLOS. Oligonucleotide array comparative genomic hybridization showed a $3.64-\mathrm{Mb}$ interstitial deletion involving most of band 10q26.12 and the proximal half of band 10q26.13; the deletion was confirmed by fluorescence in situ hybridization analysis. Parental samples were negative for the deletion.

Ophthalmologic exam was notable for pupil asymmetry, right cranial nerve III palsy, and anomalous optic nerves (right greater than left). The optic nerve in the right eye had a poorly defined margin and decreased pigmentation. There was no edema or optic atrophy in either eye. Endocrinologic workup revealed low thyroid (both free T4 and thyroid-stimulating hormone), cortisol, and testosterone levels consistent with panhypopituitarism; he was started on levothyroxine, hydrocortisone, and testosterone. His panhypopituitarism was thought to be due to hypothalamic dysfunction given a normal-

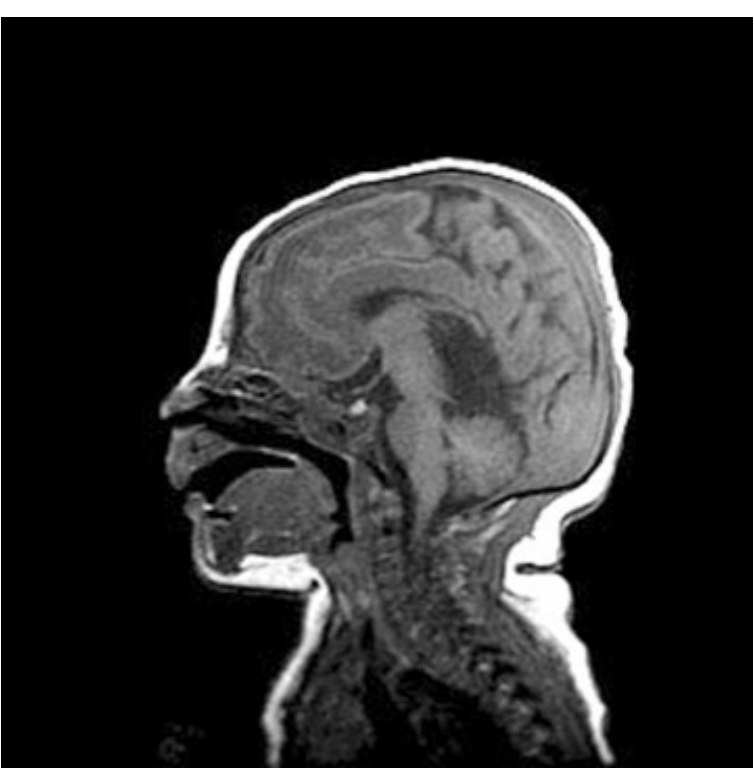

Figure 4 Midsagittal neonatal T1 image showing the pituitary (bright) 
appearing pituitary gland on MRI (Fig. 4). A gastric tube was inserted given failure to thrive and poor oral feeding during his hospital course. At 5 weeks of age, while convalescing in the intermediate nursery, he was found to be lethargic and pale; a full sepsis workup was initiated. He was transferred back to the NICU because of his clinical instability. He was noted to have excessive urine output and hypernatremia at this time and was diagnosed with diabetes insipidus for which desmopressin acetate (DDAVP) injections were initiated. Prior to discharge, he underwent initial casting of his lower extremities for his bilateral clubfeet.

He was discharged at 3 months of age with multiple medications including hydrocortisone (maintenance and instructions for stress dosing), levothyroxine, DDAVP, ferrous sulfate, and multivitamins. Close outpatient follow-up included twice-weekly appointments with his primary care physician for blood pressure and sodium-level monitoring as well as numerous subspecialty appointments. During his high-risk infant follow-up visit at 4 months of age, his developmental equivalent age was 1 to 2 months, and he was deemed to be at high risk for cerebral palsy. In the 2 months following initial discharge, he was readmitted to the hospital three times (shunt revision, Achilles tendon release, and fever). At 6 months of age he died at an outside hospital after an adrenal crisis resulted in cardiovascular collapse.

\section{DISCUSSION}

This clinical report describing the prenatal characterization of a large choroidal fissure cyst, complex postnatal course including panhypopituitarism and diabetes insipidus, diagnosis of 10q26 microdeletion, and eventual death at 6 months of age adds to the limited literature. Morioka et al described two adult cases of complex partial seizures associated with choroidal fissure cysts. $^{2} \mathrm{~A}$ wide spectrum of endocrinologic complications including growth hormone deficiency, precocious puberty, and panhypopituitarism has been described in children with intracranial arachnoid cysts. ${ }^{3}$ In a recent case report, Tas et al described a patient with bilateral choroidal fissure cysts, septo-optic dysplasia (triad of optic nerve hypoplasia, panhypopituitarism, agenesis of septum pellucidum), and infantile spasms, with shunting and cyst fenestration at 12 months. ${ }^{4}$ Similar to their case report, our patient demonstrated the severity of complications that can result from these choroid fissure cysts including panhypopituitarism and diabetes insipidus. The mechanism of the direct association of choroidal fissures and panhypopituitarism is not clear. Mohn et al speculated that endocrine disorders that occur with arachnoid cysts may be due to "simple compression" or "the expression of a complex neuroanatomical developmental anomaly and not a simple coexistence" (p. 320). ${ }^{3}$ In our case, panhypopituitarism may have been due to compression on the hypothalamus, pituitary, or both. Because in our case the pituitary appeared normal, we believe that the endocrinopathies were more likely due to hypothalamic dysfunction. In their report, Tas et al suggest that early shunting may improve outcome in these patients; however, unfortunately, this was not the case for our patient. ${ }^{4}$

It is unclear whether the clinical severity of our case was due primarily to the size of the choroidal fissure cyst or was exacerbated by the 10q26 deletion. To our knowledge, this interstitial deletion of $10 \mathrm{q} 26$ has not been previously documented in association with choroidal fissure cysts or panhypopituitarism. Indeed, our patient does not meet the phenotype of previously described cases by Irving et al. ${ }^{5}$ Patients in that report had characteristic facial features (facial asymmetry, prominent nose and nasal bridge, prominent ears, thin upper lip), growth retardation, digital abnormalities, and developmental delay. Apart from developmental delay, our patient did not share these findings. Thus, it is possible that this chromosomal deletion is an incidental finding rather than a new association. Nonetheless, it may have contributed to the adverse outcome.

Although often reported as incidental findings, choroidal fissure cysts may be associated with significant clinical complexity and unfavorable outcomes. This case underscores the need for multidisciplinary counseling and planning in prenatally diagnosed cases, meticulous evaluation and management in the neonatal period, and the need for further research in this area.

\section{REFERENCES}

1. Sherman JL, Camponovo E, Citrin CM. MR imaging of CSF-like choroidal fissure and parenchymal cysts of the brain. AJNR Am J Neuroradiol 1990;11:939-945

2. Morioka T, Nishio S, Suzuki S, Fukui M, Nishiyama T. Choroidal fissure cyst in the temporal horn associated with complex partial seizure. Clin Neurol Neurosurg 1994;96: 164-167

3. Mohn A, Schoof E, Fahlbusch R, Wenzel D, Dörr HG. The endocrine spectrum of arachnoid cysts in childhood. Pediatr Neurosurg 1999;31:316-321

4. Tas E, Tracy M, Sarco DP, Eksioglu YZ, Prabhu SP, Loddenkemper T. Septo-optic dysplasia complicated by infantile spasms and bilateral choroidal fissure arachnoid cysts. J Neuroimaging 2011;21:89-91

5. Irving $\mathrm{M}$, Hanson $\mathrm{H}$, Turnpenny $\mathrm{P}$, et al. Deletion of the distal long arm of chromosome 10; is there a characteristic phenotype? A report of 15 de novo and familial cases. Am J Med Genet A 2003;123A:153-163 